\title{
Baremación del Cuestionario de Madurez Neuropsicológica Escolar en niños residentes en Boyacá-Colombia ${ }^{1}$
}

\section{Scales of Neuropsychological Maturity Questionnaire in children living in Boyacá-Colombia}

\author{
Julian David Chinome Torres \\ Lucía Carlota Rodríguez Barreto \\ Universidad Pedagógica y Tecnológica de Colombia, Tunja, Colombia \\ Carlos Alejandro Pineda-Roa ${ }^{2}$ \\ Universidad del Norte, Barranquilla, Colombia
}

Rec (05 de Marzo de 2018) Acept (02 de Marzo de 2020)

\begin{abstract}
Resumen
El objetivo del presente trabajo fue analizar las propiedades psicométricas y obtener los baremos para población Boyacá Colombia del Cuestionario de Madurez Neuropsicológica Escolar CUMANES. Se realizó un estudio descriptivo cuantitativo de carácter psicométrico, se evaluaron 400 niños escolarizados, con edades entre 7 y 11 años, $M=8,94$ y $D E=1,812$ años. Para cuantificar la consistencia interna se estimó Alfa de Cronbach y Omega de McDonald obteniendo puntuaciones aceptables. Para identificar la estructura interna de la prueba se implementó Análisis Factorial Exploratorio el cual sugiere una dimensión del constructo y Análisis Factorial Confirmatorio permitió evidenciar buen ajuste del factor encontrado. No se hallaron diferencias estadísticamente significativas mediante la variable sexo pero si por edad. Se requieren más investigaciones en población latinoamericana que corroboren posibles diferencias culturales en la ejecución de las subpruebas del CUMANES. Finalmente, se presentan baremos para estudiantes colombianos según edad.

Palabras claves: Neuropsicología; desarrollo infantil, estudios de validación.
\end{abstract}

Correspondencia: Julian David Chinome Torres. Universidad Pedagógica y Tecnológica de Colombia juliandavid.chinome@ uptc.edu.co Tel. 3175765959

Nota de los autores

1. Este artículo es parte de los productos de un proyecto de investigación aprobado por la Dirección de Investigaciones de la Universidad Pedagógica y Tecnológica de Colombia. Código SGI- 2053.

2. Agradecimientos a Colciencias por financiación Doctorado en Psicología en Psicología. Convocatoria 727 de 2015. 


\begin{abstract}
The objective of this study was to analyze the psychometric properties and obtain the scales for the Boyacá Colombia population of the School Neuropsychological Maturity Questionnaire CUMANES. A descriptive quantitative study of psychometric character was carried out, 400 school children were evaluated, with ages between 7 and 11 years, $M=8,94$ and $S D=1,812$ years. To quantify the internal consistency, Cronbach's Alpha and McDonald's Omega were estimated obtaining acceptable scores. To identify the internal structure of the test, Exploratory Factor Analysis was implemented, which suggests a dimension of the construct and confirmatory factor analysis allowed to show good adjustment of the found factor. No statistically significant differences were found by the sex variable but by age. More research is needed in the Latin American population to corroborate possible cultural differences in the execution of the subtests of CUMANES. Finally, scales are presented for Colombian students according to age.
\end{abstract}

Keywords: Neuropsychology; child development, validation study.

\title{
Introducción
}

Según cifras de Lu, Black, y Richter (2016) para el año 2010 aproximadamente 250,4 millones de niños a nivel mundial se encontraban por debajo del desarrollo madurativo considerado como óptimo, a pesar de una disminución en esta cifra respecto a la del 2004, los autores alertan e invitan a intensificar las políticas de la primera infancia en diferentes áreas y, sobre todo en las poblaciones consideradas más vulnerables. Estas políticas se deben centrar en la prevención del riesgo en los niños más expuestos y que afectan su calidad de vida, (Engle et al., 2007; Shonkoff, Richter, van der Gaag, \& Bhutta, 2012) además, se destaca que la primera infancia es el momento oportuno para garantizar que los niños desarrollen todo su potencial madurativo, lo cual debe estar centrado en la medición y evaluación de su desempeño a través de diversas pruebas (Engle et al., 2011) especialmente las neuropsicológicas que deben tener datos normativos que correspondan a cada contexto particular.

Diversos autores han descrito el proceso de desarrollo infantil como una secuencia de etapas que se ven afectadas por variables de tipo medio ambiental y biológicas individuales, que pueden ser determinantes en edades futuras. Gottlieb, $(2002,2007)$ llamó a este proceso epigénesis probabilística, explicando que estas variables intrínsecas y extrínsecas se convierten en factores multivariados que intervienen en el desarrollo y que se expresan a través de un proceso adaptativo no lineal. Para Knickmeyer et al., (2008) y Shonkoff, Boyce, y McEwen, (2009) la calidad de las interacciones entre esos dos factores inducen cambios positivos o negativos a nivel de neurodesarrollo sobre todo en etapas sensibles como es el primer año, ya que allí pueden surgir patologías permanentes, perdurables y de difícil tratamiento.

Otras investigaciones sugieren que uno de los factores medioambientales que contribuyen de manera negativa en el desarrollo neuropsicológico infantil es la deprivación existente en los contextos en que se desenvuelven los niños, que generan alteraciones de tipo cognitivo y socio emocional que se mantienen a lo largo del tiempo desde edades tempranas hasta la adultez mayor (Pollak et al., 2010; Nelson, Furtado, Fox, \& Zeanah, 2009) son además, una dificultad prevalente en familias con ingresos bajos (Ferguson, Cassells, MacAllister, \& Evans, 2013), los niños experimentan la presencia de trastornos psiquiátricos y mayor probabilidad de sufrir comorbilidades, dificultades en el desempeño escolar, fracaso y abandono escolar y dificultades de tipo socio-económico (Ackerman \& Brown, 2006; Heckman \& Masterov, 2007; Nelson et al., 2009).

A lo largo de la infancia se evidencian procesos de neuroplasticidad, entendidos como las modificaciones anatómico-funcionales en el sistema nervioso central (SNC) cuyo objetivo se centra en la adaptación a las demandas medio ambientales que los individuos experimentan en su desarrollo madurativo (Classen, 2013; Sharma, Classen, \& Cohen, 2013) esta plasticidad, evidencia unos picos de actividad que van aproximadamente desde los 0 hacia los 5 y 6 años ya que en este tiempo existe una ganancia de peso cerebral y cambios a nivel de materia gris y blanca (Lenroot \& Giedd, 2006; Rees, Booth, \& Jones, 2016) autores como Shonkoff, (2011) y Bryck y Fisher, (2012) indican que es estas edades son importantes para aprovechar estos mecanismos de plasticidad con el fin de mitigar algunos déficits asociados a las experiencias adversas tempranas, o fortalecer las funciones cognitivas normales.

Asociado a este nivel madurativo, se asocia el término Madurez Neuropsicológica (MN) la cual se describe como el nivel de organización y desarrollo cerebral que corresponde a una edad cronológica específica, esta 
organización funcional se evidencia a través de los procesos cognitivos y conductuales que pueden ser observables y evaluados (Parra-Pulido, Rodríguez-Barreto, \& Chinome-Torres, 2016; Portellano Pérez, Mateos Mateos, Martínez Arias, Tapia Pavón, \& Granados García-Tenorio, 2000) tal como se mencionó, este proceso constituye un proceso sensible en el desarrollo madurativo, y cualquier alteración en éstas pueden incidir en la esfera cognitiva, física y emocional de los niños (Moreno \& Pérez, 2013) lo que constituye un factor crucial ya que puede ser un indicador del rendimiento académico (Ramírez, Llamas-Salguero, \& López-Fernández, 2017).

Cuando existen niveles de inmadurez neuropsicológica se encuentran dificultades en la lecto escritura y la matemáticas, también se ha relacionado con posibles déficits comportamentales en la infancia o la adultez, no obstante ésta no se relaciona con una disfunción neurológica profunda, déficits sensoriales o daño cerebral adquirido, por el contrario, se caracteriza por su difícil diagnóstico y se corrobora con la evaluación neuropsicológica de tipo no invasivo, ya que estudios de neuroimagen no detectan anomalías fácilmente. (Enseñat \& Picó, 2011 ; Portellano, 2005; Portellano, 2007; Rubia, Alegría, \& Brinson, 2014; Warnke, 2012).

Se han descrito factores etiológicos asociadas a la inmadurez neuropsicológica, tales como los problemas de prematuridad o bajo peso al nacer, (Dundar, Béteille, Riboud \& Deolalikara, 2014; Lowe et al., 2014; Parra Pulido, Rodríguez Barreto, \& Chinome Torres, 2015) con las funciones metabólicas cerebrales y los trastornos a nivel pre, peri o postnatales (Lavrik, Domitrak \& Shprakh, 2011; Pearce, Manna, Singha \& Sayersa, 2014) si los problemas de inmadurez persisten pueden asociarse a los signos neurológicos blandos, (Matute, Rosselli \& Ardila, 2010) por esto, es prioritario realizar un seguimiento constante a los niños que pueden tener algún riesgo de dificultades a nivel neurocognitivo sobre todo, al momento de ingresar en la escolaridad formal (Benítez, Bringas, Jiménez-Morales, \& Macías, 2015).

Según cifras del Departamento Administrativo Nacional de Estadística (DANE), (2011) en Colombia, se estima que existen dificultades asociadas a habilidades neurocognitivas que tienen escasa demanda en consulta profesional en la infancia, lo cual incide en el desempeño de los estudiantes ya sea en su desarrollo cotidiano o en sus habilidades académicas. Dado que los niños pasan desapercibidos debido a que la problemática generalmente se detecta hasta el inicio de la edad escolar, dejando como resultado, un incremento en los costos de intervención, déficits en la implementación de programas de rehabilitación y mayor demanda de consulta profesional (Matute, et al., 2010). Lo anterior hace necesario que se cuente con evaluaciones que sean sensibles a la detección de dificultades en la madurez neuropsicológica en Colombia, para evitar a futuro que se perpetúen dichas problemáticas.

Asociado a lo anterior, es indispensable contar con las herramientas diagnósticas adecuadas, la valoración en la madurez neuropsicológica permitirá dar un abordaje adecuado para cada caso específico, y hace de este constructo neuropsicológico un factor importante para disminuir dificultades a largo plazo, como, por ejemplo, en la detección oportuna de trastornos específicos de aprendizaje (TEA) que tienen como consecuencia a largo plazo el fracaso y abandono escolar.

En la actualidad existen test neuropsicológicos, que permiten evaluar diversos constructos neuropsicológicos, no obstante, muchos de ellos son adaptaciones de test neuropsicológicos para adultos, elaborados de manera general y en una población en particular, lo que genera resultados sesgados y diferencias significativas entre las poblaciones donde se aplica el test (Portellano \& García, 2014).

Al respecto, Casari y Oros, (2013) invitan a los investigadores a tener precaución en la interpretación de las pruebas que no cuentan con una estandarización en la población donde ha sido aplicada y hacen énfasis en la baremación de pruebas neuropsicológicas, ya que esto permitirá hacer una aproximación a un diagnóstico adecuado en los lugares donde se lleven a cabo las valoraciones, las influencias socioculturales pueden ser determinantes y son motivo de sesgo en el abordaje de la valoración neurocognitiva.

En Colombia, para Ardila y Ostrosky, (2012) las pruebas neuropsicológicas son escasas y a veces insuficientes, indican que, algunas de estas no cuentan con los parámetros psicométricos para dar una aproximación diagnóstica adecuada, lo cual no permitiría realizar valoraciones validas, que minimicen el sesgo sociocultural, afectando directamente al paciente evaluado, ya que muchas de las pruebas no cuentan con baremos en la población colombiana.

El uso de pruebas para evaluar población con o sin patología requiere de instrumentos confiables adaptados específicamente para el contexto y la población que se pretende evaluar. (Squire y Shimamura citado por Pérez 2009). El CUMANES fue diseñado y validado en España con un número total de 766 participantes, con edades comprendidas entre los 7 y 11 años, provenientes de 4 centros de carácter público y 4 de carácter privado. Los 
coeficientes de fiabilidad como consistencia interna de las pruebas a partir del estadístico Alfa de Cronbach obtenidos fueron: Comprensión Audioverbal= 0,67, Comprensión de Imágenes= 0,80, Fluidez (incluye las 2 pruebas de fluidez) $=0,64$, Leximetría (Solo incluye comprensión lectora) $=0,61$, Escritura Audiognósica $=0,81$, Visopercepción=0,85, Memoria Verbal=0,68, Memoria Visual=0,63 y Ritmo=0,83.

Para el caso de la validez de constructo, Portellano, Mateos y Martínez-Arias (2012) realizaron Análisis Factorial Exploratorio (AFE) y Análisis Factorial Confirmatorio (AFC), se sometió a prueba un modelo basado en un solo factor llamado Índice de Desarrollo Neuropsicológico (IDN), el cual evidenció valores de ajuste aceptable, lo que permitió obtener una puntuación global a partir de todas las sub escalas del CUMANES, este análisis arrojó un valor ponderado, correspondiente a los pesos factoriales del modelo de Análisis Factorial Confirmatorio (AFC) de un solo factor, los valores negativos en las escalas de Función Ejecutiva se debe a que a mayor edad estas puntuaciones decrecen.

El cuestionario presenta los pesos factoriales y los pesos factoriales ajustados por cada sub escala, los cuales son útiles en la obtención del IDN, ya que se calcula a partir de la suma de los puntajes de transformación, los cuales son la multiplicación de las puntuaciones directas por los pesos factoriales transformados, y se presentan en los baremos en el recuadro de puntuación transformada (Portellano, Mateos y Martinez-Arias, 2012, p. 127). Se encuentran baremos con respecto a la variable edad debido a que no se encontraron diferencias significativas entre las variables sexo.

El CUMANES (Portellano, Mateos, \& Martínez-Arias, 2012) puede ser aplicado a niños entre los 7 y 11 años de edad y es la continuidad del CUMANIN, ha sido calificado por Zamora Crespo \& García-Navarro, (2014) como uno de los cuestionarios desarrollados específicamente para la población infantil, y es en la actualidad una de las más utilizadas en la población pediátrica, y por la población hispano-hablante, (Benítez et al., 2015) sin embargo, carece de datos normativos para la población Colombiana, lo cual sugiere la importancia de la obtención de baremos en la población a quien va dirigida esta evaluación en particular, por último, se realizó una búsqueda en bases de datos, sobre las baremaciones que ha tenido el CUMANES, en SCOPUS, APA PsycNET, EBSCOhost, DOAJ, Science Direct, Scielo y Redalyc, sin encontrar ningún estudio asociado.

El objetivo principal en la presente investigación fue analizar las propiedades psicométricas y obtener los baremos del CUMANES, (Portellano et al., 2012) en una muestra de niños y niñas de 7 a 11 años, residentes en el departamento de Boyacá Colombia.

\section{Método}

\section{Tipo de estudio}

Se realizó una investigación de tipo descriptivo y cuantitativo, (Hernandez Sampieri, Fernandez Collado, \& Baptista Lucio, 2010). El diseño de la investigación fue un estudio no experimental de carácter psicométrico ya que se desea obtener los baremos para una población boyacense en particular, sin realizar ninguna manipulación intencional de variables (Tornimbeni et al., 2008).

\section{Participantes}

La muestra total estuvo conformada por 400 niños (as) con edades comprendidas entre los 7 y 11 años, la media de la edad fue de 107,28 meses (8,94 años), con una desviación típica de 16,812 meses (1,401 años) ( $M E=8,94$ años; $D E=1,401$ años), con un rango de 7,1 años a 11,10 años. De los cuales 197 participantes eran mujeres $(49,3 \%)$ y 203 hombres $(50,7 \%)$ (Tabla 1$)$, escolarizados en 5 instituciones de carácter público y en 5 privadas, de 4 ciudades del departamento de Boyacá de las provincias Centro y Tundama.

Para esta investigación los criterios de inclusión fueron: (a) tener edades comprendidas entre los 7 y 11 años al momento de la ejecución de la investigación (b) saber leer y escribir (c) tener autorización por parte de sus padres y/o representante legal para la participación de este estudio con la firma del consentimiento informado y (d) que el participante no tuviera alguna limitación motriz o sensorial que impidiera realizar la prueba. 


\section{Instrumentos}

El CUMANES se aplica de manera individual a niños con edades entre los 7 y 11 años, su duración oscila entre los 40 y 50 minutos, su finalidad es evaluar de forma global el desarrollo neuropsicológico en el ámbito clínico o educativo. La mayoría de los ítems son independientes, obteniendo una puntuación directa a partir de la suma de las respuestas correctas con modalidad dicotómicas o politómicas. Cuenta con baremos originales para niños de España, descritos en modo de decatipos para cada una de las pruebas y percentiles de la puntuación total en cada grupo de edad. La prueba tiene 12 sub escalas y 6 áreas, dentro del lenguaje se encuentran las sub escalas de (comprensión audio-verbal, comprensión de imágenes, fluidez fonológica, fluidez semántica, leximetría [comprensión y velocidad lectora] y escritura audiognósica), las otras escalas son visopercepción, funcionamiento ejecutivo, (tiempo y errores), memoria (verbal y visual) y ritmo. A partir del desempeño en cada escala se obtiene una puntuación global que resume el nivel de madurez neuropsicológica (Índice de Desarrollo Neuropsicológico), cuenta también con escala de lateralidad, que no es necesaria para la obtención del IDN.

\section{Procedimiento}

1. Contacto con las Instituciones Educativas: Se realizó el contacto pertinente con las instituciones educativas en la modalidad privada y pública de los municipios seleccionados previamente, con el fin de presentar la propuesta de investigación, en la socialización se acordaron los tiempos de la aplicación y la selección de espacios adecuados para cada aplicación teniendo en cuenta los factores medioambientales evitando posibles distractores.

2. SocializACión A PADRES DE FAMILIA: Se estableció contacto con los padres de los participantes a quienes se les socializó el procedimiento y dinámicas del proyecto, también se informó los objetivos, los derechos como participantes, deberes como investigadores y las normas éticas que rigen la investigación. Se resolvieron algunas dudas a los padres de familia, y se procedió con la firma del consentimiento informado.

3. CRONogRAMA DE APLICACIÓN: Teniendo en cuenta los horarios asignados en cada institución se diseñó un cronograma para la aplicación de los Cuestionarios.

4. Aplicación del Cuestionario: Se llevó a cabo con los protocolos originales y de forma individual, en un espacio y tiempo apropiado en donde solo se encontraba el investigador y el participante. Basados en las recomendaciones de los autores, se tuvieron en cuenta las normas técnicas de aplicación del CUMANES.

5. Entrega de Resultados: Se entregaron los informes a cada uno de los padres de familia, previa citación en cada una de las instituciones educativas, el informe incluía una serie de recomendaciones y estrategias en casa para promover el desarrollo neurocognitivo del niño.

\section{Consideraciones éticas}

Esta investigación cuenta con el aval del comité de ética de la Vicerrectoría de Investigaciones y Extensión (VIE) de la Universidad Pedagógica y Tecnológica de Colombia. El consentimiento informado incluye los lineamientos de la Ley 1090 de 2006 en el literal D, del artículo 25, los padres de familia o tutores legales lo firmaron en la reunión de socialización del proyecto, respondiendo previamente a las inquietudes. También se tomaron algunos lineamientos de la Resolución 8430 de 1993 donde se tienen cuenta las normas para la investigación en salud, calificándose como investigación sin riesgo, ya que no involucró la manipulación de variables biológicas, fisiológicas, sociales o de cambios de conducta intencionado.

\section{Análisis estadístico}

Los análisis se presentan en términos de frecuencias y porcentajes para variables categóricas y medias y desviación estándar para variables de intervalo. Para cuantificar la consistencia interna se calcula el alfa de Cronbach y omega de McDonald. Para identificar la estructura interna de la prueba se implementa Análisis Factorial Exploratorio AFE y Análisis Factorial Confirmatorio AFC. Para conocer la bondad del ajuste de los datos al modelo teórico se calcularon la prueba chi cuadrado de Satorra-Bentler ( $\chi 2$ S-B), con grados de libertad gl, valor de probabilidad p y los coeficientes Root Mean Square Error of Approximation, (RMSEA), intervalo de 
confianza del 90\%, Comparative Fit Index, (CFI), Non Normed Fit Index (NNFI) y Standardized Mean Square Residual, (SRMS). Con el fin de obtener un buen ajuste, se esperan valores de RMSEA y SRMS $<0.08$ (Browne \& Cudeck, 1993); para AGFI, CFI, NNFI valores $>0.89$ y cociente $\chi 2 / g 1$ menor a 3 , considerado óptimo. Los datos fueron analizados a través del programa estadístico SPSS en su versión 22.0 y AFC se calculó mediante Lisrel versión 8.8 (Jöreskog \& Sörbom, 2012). Luego de verificar las propiedades psicométricas se procedió a identificar diferencias significativas según sexo y edad mediante estadísticos t y $\mathrm{F}$ con magnitud del efecto, previa consideración de los supuestos de tales estadísticos.

\section{Resultados}

Se presenta inicialmente la composición total de muestra por sexo y edad, seguida de los descriptivos de cada una de las subescalas discriminados por la edad del participante. A continuación, se muestran los valores de consistencia interna. Por último, los resultados de validez de constructo mediante Análisis Factorial Exploratorio AFE y Análisis Factorial Confirmatorio AFC. Los baremos se calcularon por edad según se hayan encontrado diferencias significativas y estos aparecen en Anexos.

Tabla 1. Distribución de la muestra en función de la edad y el sexo

\begin{tabular}{ccccccc} 
& \multicolumn{3}{c}{ Sexo } & \multicolumn{2}{c}{ Total } \\
\cline { 2 - 5 } Edad años & \multicolumn{2}{c}{ Masculino } & \multicolumn{3}{c}{ Femenino } & \multicolumn{2}{c}{ n } & $\%$ \\
\cline { 2 - 5 } & $\mathrm{N}$ & $\%$ & $\mathrm{~N}$ & $\%$ & 77 & 19,4 \\
8 & 41 & 53,2 & 36 & 56,8 & 96 & 24 \\
9 & 44 & 45,8 & 52 & 54,2 & 75 & 18,8 \\
10 & 40 & 53,3 & 35 & 46,7 & 76 & 19 \\
11 & 39 & 51,3 & 37 & 48,7 & 76 & 18,8 \\
Total & 39 & 51,3 & 37 & 46,7 & 400 & 100 \\
\hline
\end{tabular}

Fuente: Elaboración Propia

La tabla 1 muestra una distribución balanceada en la composición de la muestra por edad y sexo. No se encontraron diferencias significativas en el promedio de edad entre hombres y mujeres, $\left(\mathrm{t}_{398}=0,154 ; \mathrm{p}=0,87\right.$, $\mathrm{d}=0,02$ ). Por otro lado, la tabla 2 muestra que a medida que la edad aumenta, se pueden apreciar niveles promedio ascendentes en cada una de las 12 subpruebas del CUMANES. 
Tabla 2. Estadísticos descriptivos de las pruebas del CUMANES

\begin{tabular}{|c|c|c|c|c|c|}
\hline Edad & Prueba & Min & Max & $M$ & $D T$ \\
\hline \multirow{13}{*}{7 años } & Comprensión audioverbal & 0 & 9 & 4,58 & 2,09 \\
\hline & Comprensión imágenes & 2 & 17 & 8,85 & 3,57 \\
\hline & Fluidez fonológica & 1 & 13 & 6,8 & 2,41 \\
\hline & Fluidez semántica & 4 & 24 & 12 & 3,7 \\
\hline & Comprensión lectora & 1 & 8 & 4,09 & 1,39 \\
\hline & Escritura audiognósica & 0 & 12 & 4,71 & 2,78 \\
\hline & Visopercepción & 2 & 28 & 15,29 & 5,57 \\
\hline & Función ejecutiva tiempo & 28 & 485 & 222,83 & 71,87 \\
\hline & Función ejecutiva error & 0 & 13 & 1,38 & 2,07 \\
\hline & Memoria verbal & 8 & 28 & 18,1 & 3,53 \\
\hline & Memoria visual & 4 & 13 & 8,58 & 2,16 \\
\hline & Ritmo & 0 & 11 & 1,44 & 1,82 \\
\hline & Indice de desarrolllo neuropsicológico & 28 & 84 & 52,51 & 12,75 \\
\hline Edad & Prueba & Min & Max & $M$ & $D T$ \\
\hline \multirow{13}{*}{8 años } & Comprensión audioverbal & 0 & 10 & 5,07 & 2,63 \\
\hline & Comprensión imágenes & 1 & 19 & 10,15 & 3,91 \\
\hline & Fluidez fonológica & 2 & 15 & 8,03 & 2,48 \\
\hline & Fluidez semántica & 3 & 20 & 13,36 & 3,21 \\
\hline & Comprensión lectora & 0 & 8 & 4,48 & 1,73 \\
\hline & Escritura audiognósica & 0 & 14 & 6,12 & 3,37 \\
\hline & Visopercepción & 0 & 33 & 18,27 & 6,59 \\
\hline & Función ejecutiva tiempo & 22,6 & 409 & 197,54 & 67,67 \\
\hline & Función ejecutiva error & 0 & 12 & 1,06 & 1,69 \\
\hline & Memoria verbal & 10 & 28 & 19,75 & 3,98 \\
\hline & Memoria visual & 2 & 14 & 9,72 & 1,87 \\
\hline & Ritmo & 0 & 16 & 2,6 & 3,31 \\
\hline & Indice de desarrolllo neuropsicológico & 36 & 104 & 61,11 & 15 \\
\hline Edad & Prueba & Min & Max & $M$ & DT \\
\hline \multirow{13}{*}{9 años } & Comprensión audioverbal & 1 & 10 & 5,17 & 2,22 \\
\hline & Comprensión imágenes & 2 & 17 & 11,84 & 3,11 \\
\hline & Fluidez fonológica & 3 & 16 & 8,89 & 2,79 \\
\hline & Fluidez semántica & 9 & 27 & 14,44 & 3,2 \\
\hline & Comprensión lectora & 2 & 8 & 5,04 & 1,41 \\
\hline & Escritura audiognósica & 0 & 13 & 7 & 3,07 \\
\hline & Visopercepción & 5 & 33 & 19,2 & 5,67 \\
\hline & Función ejecutiva tiempo & 100 & 255 & 174,42 & 43,47 \\
\hline & Función ejecutiva error & 0 & 13 & 1,01 & 1,84 \\
\hline & Memoria verbal & 10 & 27 & 19,72 & 4,31 \\
\hline & Memoria visual & 5 & 14 & 9,82 & 2,12 \\
\hline & Ritmo & 0 & 15 & 3,32 & 3,33 \\
\hline & Índice de desarrollo neuropsicológico & 40 & 96 & 67,18 & 12,19 \\
\hline
\end{tabular}




\begin{tabular}{|c|c|c|c|c|c|}
\hline Edad & Prueba & Min & Max & $M$ & $D T$ \\
\hline \multirow{13}{*}{10 años } & Comprensión audioverbal & 0 & 10 & 4,4 & 2,16 \\
\hline & Comprensión imágenes & 2 & 16 & 11,63 & 3,18 \\
\hline & Fluidez fonológica & 4 & 16 & 8,89 & 2,73 \\
\hline & Fluidez semántica & 5 & 22 & 14,5 & 3,07 \\
\hline & Comprensión lectora & 0 & 8 & 4,68 & 1,65 \\
\hline & Escritura audiognósica & 1 & 14 & 7,57 & 3,58 \\
\hline & Visopercepción & 5 & 36 & 19,77 & 6,1 \\
\hline & Función ejecutiva tiempo & 62 & 311 & 164,13 & 55,32 \\
\hline & Función ejecutiva error & 0 & 6 & 0,86 & 1,32 \\
\hline & Memoria verbal & 10 & 30 & 20,46 & 4,6 \\
\hline & Memoria visual & 5 & 15 & 10,38 & 2,2 \\
\hline & Ritmo & 0 & 20 & 3,57 & 4,24 \\
\hline & Índice de desarrollo neuropsicológico & 38,6 & 98 & 68,05 & 13,68 \\
\hline Edad & Prueba & Min & Max & $M$ & $D T$ \\
\hline \multirow{13}{*}{11 años } & Comprensión audioverbal & 1 & 10 & 5,38 & 2,07 \\
\hline & Comprensión imágenes & 2 & 18 & 12,61 & 3,73 \\
\hline & Fluidez fonológica & 4 & 19 & 10,42 & 2,75 \\
\hline & Fluidez semántica & 7 & 28 & 16,43 & 3,54 \\
\hline & Comprensión lectora & 1 & 8 & 4,46 & 1,41 \\
\hline & Escritura audiognósica & 1 & 20 & 8,9 & 3,77 \\
\hline & Visopercepción & 13 & 35 & 21,6 & 5,01 \\
\hline & Función ejecutiva tiempo & 75 & 232 & 145,47 & 33 \\
\hline & Función ejecutiva error & 0 & 3 & 0,61 & 0,81 \\
\hline & Memoria verbal & 10 & 29 & 22,19 & 4,18 \\
\hline & Memoria visual & 6 & 15 & 10,68 & 2,22 \\
\hline & Ritmo & 0 & 17 & 5,73 & 4,83 \\
\hline & Índice de desarrollo neuropsicológico & 47 & 114 & 78,32 & 12,84 \\
\hline
\end{tabular}

Fuente: Elaboración propia

En general la consistencia interna fue aceptable, con valores Alfa y Omega por encima de 0,60 (Kline, 2012), excepto para dos de las subescalas (tabla 3). No obstante, dado los problemas del Alfa de Cronbach, es muy probable que el Omega de McDonald sea una mejor estimación de la confiabilidad, dado que no exige el supuesto de tau-equivalencia y es más robusto al incumplimiento de supuestos en relación con el Alfa de Cronbach (Revelle \& Zinbarg, 2009; Sijtsma, 2009). 
Alejandro Pineda-Roa

Tabla 3. Resultados de confiabilidad por consistencia interna de las subescalas del CUMANES

\begin{tabular}{ccc}
\hline Prueba & Alfa de Cronbach & Omega de Mcdonald \\
\hline Comprensión audioverbal & 0,60 & 0,73 \\
Comprensión de imágenes & 0,79 & 0,83 \\
Fluidez (2 pruebas) & $0,62^{*}$ & -- \\
Leximetría & 0,42 & 0,56 \\
Escritura audiognósica & 0,72 & 0,72 \\
Visopercepción & 0,80 & 0,80 \\
Memoria verbal & 0,74 & 0,70 \\
Memoria visual & 0,42 & 0.51 \\
Ritmo & 0,80 & 0,92 \\
\hline
\end{tabular}

Fuente: Elaboración propia

*Lambda 4 de Guttman con dos partes

La tabla 4 muestra las intercorrelaciones de las distintas subpruebas, se encontraron correlaciones estadísticamente significativas con $\mathrm{p}<0,001$ y $\mathrm{p}<0,05$, con valores positivos y negativos que oscilan entre 0,095 y 0,442 y $-0,373$ y $-0,57$ respectivamente. De igual manera, se hallaron puntuaciones negativas en las escalas relacionadas a función ejecutiva, exceptuando la correlación entre la escala de funciones ejecutiva error y función ejecutiva tiempo relacionada con una relación inversa entre edad y el tiempo empleado en la ejecución de la tarea. Esto evidencia que cada prueba tiende a medir elementos de una misma construcción teórica como lo es madurez neuropsicológica.

Tabla 4. Correlaciones entre las puntuaciones e las pruebas

\begin{tabular}{|c|c|c|c|c|c|c|c|c|c|c|c|c|}
\hline & 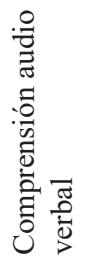 & 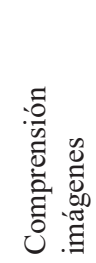 & 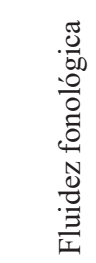 & 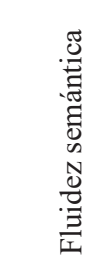 & 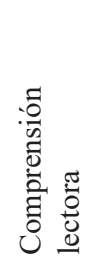 & 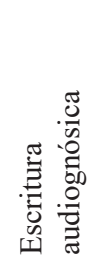 & 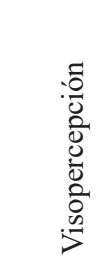 & 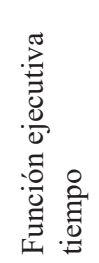 & 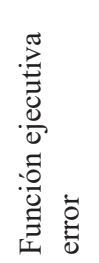 & 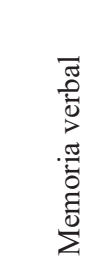 & 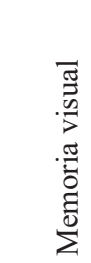 & 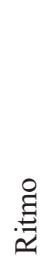 \\
\hline $\begin{array}{l}\text { Comprensión } \\
\text { Audioverbal }\end{array}$ & 1 & & & & & & & & & & & \\
\hline Comprensión imágenes &, $287 * *$ & 1 & & & & & & & & & & \\
\hline Fluidez fonológica &, $214 * *$ &, $404 * *$ & 1 & & & & & & & & & \\
\hline Fluidez semántica &, $253 * *$ &, $373 * *$ &, $442 * *$ & 1 & & & & & & & & \\
\hline Comprensión lectora &, $547 * *$ &, $362 * *$ & ,286** &, $246 * *$ & 1 & & & & & & & \\
\hline Escritura audiognósica &, $281 * *$ &, $385^{* *}$ &, $373 * *$ &, $344 * *$ &, $382 * *$ & 1 & & & & & & \\
\hline Visopercepción &, $208 * *$ & ,095 &, $212 * *$ &, $249 * *$ &, $196^{* *}$ &, $338 * *$ & 1 & & & & & \\
\hline $\begin{array}{l}\text { Función ejecutiva } \\
\text { tiempo }\end{array}$ &,$- 223 * *$ &,$- 262 * *$ &,$- 330 * *$ &,$- 373 * *$ &,$- 281 * *$ &,$- 289 * *$ &,$- 216^{* *}$ & 1 & & & & \\
\hline Función ejecutiva error &, $161^{* *}$ &,$- 117^{*}$ &,- 074 &,- 057 &,$- 121^{*}$ &,$- 187^{* *}$ &,$- 154 * *$ &, $116^{*}$ & 1 & & & \\
\hline Memoria verbal &, $241 * *$ &, $328 * *$ &, $354 * *$ &, $338 * *$ &, $277^{* *}$ &, $285 * *$ &, $196 * *$ &,$- 210 * *$ &,- 060 & 1 & & \\
\hline Memoria visual &, $169 * *$ &, $284 * *$ &, $318^{* *}$ &, $322 * *$ &, $240 * *$ &, $202 * *$ &, $167 * *$ &,$- 204 * *$ &,- 086 &, $307 * *$ & 1 & \\
\hline Ritmo &, $287 * *$ &, $359 * *$ &, $292 * *$ &, $273 * *$ &, $221 * *$ &, $378 * *$ &, $274 * *$ &,$- 252 * *$ &,$- 132 * *$ &, $264 * *$ &, $181 * *$ & 1 \\
\hline
\end{tabular}

Fuente: Elaboración propia

**. La correlación es significativa en el nivel 0,01 (2 colas).

* La correlación es significativa en el nivel 0,05 (2 colas). 
Para proceder a realizar un Análisis Factorial Exploratorio AFE, KMO $(0,85)$ y test de esfericidad de Bartlet $(1001,09 ; p=0,000)$ permitieron establecer la factibilidad de esta técnica. Un análisis paralelo de Horn (1965) permitió retener un único factor el cual explicó un $32 \%$ de varianza. El factor encontrado puede denominarse índice de desarrollo neuropsicológico. La tabla 5 confirma un ajuste adecuado para modelo compuesto por un factor, el cual arrojó índices razonablemente buenos.

Tabla 5. Análisis factorial confirmatorio del CUMANES (solución estandarizada)

\begin{tabular}{lllllllll}
\hline Modelo & $\chi_{\text {S-B }}^{2}$ & gl & GFI & NNFI & CFI & SRMR & RMSEA & IC 90 \% \\
\hline Un factor & $183.91 *$ & 54 & 0.96 & 0.91 & 0.93 & 0.05 & 0.07 & {$[0.06,0.09]$} \\
\hline
\end{tabular}

Fuente: elaboración propia.

$\mathrm{IC}=$ Intervalo de confianza; $\chi_{\mathrm{S}-\mathrm{B}}^{2}$ : Chi cuadrado de Satorra Bentler; gl: Grados de libertad; RMSEA: Root Mean Square Error of Approximation;GFI Goodness Fitnes Index; CFI: Comparative Fit Index. NNFI: Non-Normed of Fit Index. SRMR: Standard Residual Mean Root $* p<0.05$.

A continuación, se presentan los análisis t-student y Análisis de Varianza de una vía con el fin detectar diferencias entre las variables sexo y edad, para determinar el criterio de construcción de los baremos. Todos los puntajes totales del índice de madurez neuropsicológica se distribuyeron normalmente (K.S. $=0,20, p>0,05$ ). Por tanto, se procedió a calcular pruebas t para detectar diferencias estadísticamente significativas por sexo y Anova de un Factor para la edad.

Tabla 6. Prueba t-student para en el Índice de Desarrollo Neuropsicológico según sexo

\begin{tabular}{llrrrrr}
\hline & Sexo & $\mathrm{N}$ & Media & DE & $p$ & $d$ \\
\hline Índice de desarrollo Neuropsicológico & Masculino & 203 & 96,0493 & 14,76239 & 0,845 & 0.01 \\
& Femenino & 197 & 95,7519 & 15,64718 & 0,845 & \\
\hline
\end{tabular}

Fuente: elaboración propia.

Los resultados mostrados en la tabla 6 no evidencian diferencias estadísticamente significativas, por esta razón no es necesario realizar baremos para la variable sexo. Por su parte, el Anova de un factor evidenció diferencias estadísticamente significativas según edad, $\mathrm{F}_{(4,389)}=38,38, \mathrm{p}=0,00, \eta^{2}=0.28$ ). En consecuencia, se realizó un análisis post-hoc con prueba de Bonferroni para determinar entre qué grupos había diferencias en el desempeño de la prueba basado según la edad.

\section{Discusión}

El objetivo de la investigación fue analizar las propiedades psicométricas y obtener los baremos del Cuestionario de Madurez Neuropsicológica Escolar (CUMANES) para la población de Boyacá Colombia, esto, para evitar sesgos interpretativos al usar normas interpretativas ajenas al contexto de evaluación (Ardila \& Ostrosky, 2012; Portellano \& García, 2014).

Según los resultados obtenidos en la presente investigación, el CUMANES, arroja datos de consistencia interna similares a los hallados por Portellano et al., (2012) en su prueba original, considerados como aceptables (Kline, 2013), exceptuando en las sub escalas de leximetría y memoria visual. Estas dos sub escalas en la prueba original también obtuvieron puntuaciones Alfa considerados como aceptables leximetría $(0,61)$ y memoria visual $(0,63)$. Se utilizaron dos estadísticos de fiabilidad, ya que se siguieron las sugerencias de Green y Yang, (2009) con respecto al uso del Alfa, utilizando también el Omega de McDonald, quien mostró mejores puntuaciones, no obstante, las sub escalas de leximetría y memoria visual obtuvieron puntuaciones por debajo de lo aceptable, lo cual puede tener relación con la dificultad creciente de las pruebas. 
Tabla 7. Análisis de varianza post-hoc en el índice de desarrollo neuropsicológico según edad

\begin{tabular}{|c|c|c|c|c|c|c|}
\hline \multirow[b]{2}{*}{ (I) EDAD AÑOS } & & \multirow{2}{*}{$\begin{array}{l}\text { Diferencia de } \\
\text { medias (I-J) }\end{array}$} & \multirow[b]{2}{*}{ Error estándar } & \multirow[b]{2}{*}{$p$} & \multicolumn{2}{|c|}{ IC $95 \%$} \\
\hline & & & & & Límite inferior & Límite superior \\
\hline \multirow[t]{4}{*}{7} & 8 & $-8,60226^{*}$ & 2,08619 & 000 & $-14,4919$ & $-2,7127$ \\
\hline & 9 & $-14,67213^{*}$ & 2,18980 &, 000 & $-20,8543$ & $-8,4900$ \\
\hline & 10 & $-15,54261^{*}$ & 2,18259 &, 000 & $-21,7044$ & $-9,3809$ \\
\hline & 11 & $-25,81103^{*}$ & 2,18259 &, 000 & $-31,9728$ & $-19,6493$ \\
\hline \multirow[t]{4}{*}{8} & 7 & $8,60226^{*}$ & 2,08619 &, 000 & 2,7127 & 14,4919 \\
\hline & 9 & $-6,06987^{*}$ & 2,08619 &, 038 & $-11,9595$ &,- 1803 \\
\hline & 10 & $-6,94034^{*}$ & 2,07862 & 009 & $-12,8086$ & $-1,0721$ \\
\hline & 11 & $-17,20876^{*}$ & 2,07862 &, 000 & $-23,0770$ & $-11,3405$ \\
\hline \multirow[t]{4}{*}{9} & 7 & $14,67213^{*}$ & 2,18980 &, 000 & 8,4900 & 20,8543 \\
\hline & 8 & $6,06987^{*}$ & 2,08619 &, 038 & , 1803 & 11,9595 \\
\hline & 10 &,- 87047 & 2,18259 & 1,000 & $-7,0322$ & 5,2913 \\
\hline & 11 & $-11,13889^{*}$ & 2,18259 &, 000 & $-17,3006$ & $-4,9771$ \\
\hline \multirow[t]{4}{*}{10} & 7 & $15,54261^{*}$ & 2,18259 &, 000 & 9,3809 & 21,7044 \\
\hline & 8 & $6,94034^{*}$ & 2,07862 &, 009 & 1,0721 & 12,8086 \\
\hline & 9 &, 87047 & 2,18259 & 1,000 & $-5,2913$ & 7,0322 \\
\hline & 11 & $-10,26842^{*}$ & 2,17535 &, 000 & $-16,4097$ & $-4,1271$ \\
\hline \multirow[t]{4}{*}{11} & 7 & $25,81103^{*}$ & 2,18259 &, 000 & 19,6493 & 31,9728 \\
\hline & 8 & $17,20876^{*}$ & 2,07862 &, 000 & 11,3405 & 23,0770 \\
\hline & 9 & $11,13889^{*}$ & 2,18259 &, 000 & 4,9771 & 17,3006 \\
\hline & 10 & $10,26842^{*}$ & 2,17535 &, 000 & 4,1271 & 16,4097 \\
\hline
\end{tabular}

Fuente: Elaboración propia. * La diferencia de medias es significativa en el nivel 0.05 .

Análisis de post-hoc de la tabla 7 sugiere la elaboración de baremos por todos los grupos etarios entre 7 y 11 años.

Por su parte, los coeficientes de correlación mostraron puntuaciones similares a lo hallado por Portellano et al. (2012). Además, la mayoría de las correlaciones superan el valor mínimo aceptable para considerarse una asociación importante, según Kaplan \& Saccuzzo (2006).

El presente estudio probó los tres modelos sugeridos por Portellano, Mateos y Martínez (2012). No obstante, luego de varios análisis factoriales, no se encontró apoyo empírico para los modelos de dos y tres factores. El análisis factorial exploratorio y el Análisis Paralelo arrojaron un único factor. Por tanto, se decide optar por un único factor debido a que posee un ajuste aceptable, permitiendo obtener un índice global (IDN) como único factor encontrado a partir de AFE y confirmado mediante AFC a partir de las sub pruebas del cuestionario (Portellano, Mateos \& Martínez, 2012, p. 87). A pesar de obtener un valor $p$ significativo de la prueba Chi cuadrado en el AFC, el cual indicaría que los datos no ajustan al modelo unifactorial, es importante tener presente la sensibilidad de $\chi^{2}$ en muestras grandes. Por tanto, se suele analizar la razón entre $\chi^{2} / \mathrm{g}$. Con base en dicha razón, es posible afirmar que el ajuste fue aceptable $\left(\chi^{2} / \mathrm{gl}=3,40\right)$.

A partir del análisis de las propiedades psicométricas arrojadas se concluye que el CUMANES cuenta con una consistencia interna aceptable en las sub escalas; a través del análisis factorial se encontró un modelo de un único factor, el cual es aplicable para el constructo de madurez neuropsicológica, lo que permite inferir unidimensionalidad en el cuestionario y sus subescalas cumpliendo con todos los supuestos estadísticos catalogados como aceptables (Browne y Cudeck, 1993), de manera similar a la prueba original para infantes (Portellano, Mateos, Martínez, Granados \& Tapia 2009). 
No se evidenciaron diferencias significativas en la variable sexo, pero sí se hallaron diferencias significativas en la variable edad y la magnitud del efecto fue baja según los criterios de Ferguson et al. (2009), tales diferencias son esperable en términos madurativos, por lo tanto, a partir de los resultados se ve la necesidad de realizar la obtención de los baremos a partir de la variable edad, como criterio diferenciador (ver baremos en Anexo).

Se recomienda a los psicólogos colombianos implementar los baremos de la presente investigación en la evaluación de la madurez neuropsicológica de niños colombianos con características similares a los de la presente muestra. El presente estudio constituye un aporte al conocimiento del constructo madurez neuropsicológica en niños colombianos en los cuales se evidenció un desempeño neuropsicológico muy similar en la mayoría de las subpruebas en todos los grupos etarios, quizá en un mundo globalizado poca es la influencia que estos datos pueden atribuir a diferencias culturales en términos madurativos. Futuros investigaciones podrán corroborar los resultados aquí encontrados con poblaciones más representativas de la niñez colombiana y latinoamericana.

\section{Referencias}

Ackerman, B. P., \& Brown, E. D. (2006). Income poverty, poverty co-factors, and the adjustment of children in elementary school. Advances in Child Development and Behavior, 34, 91-129. https://doi.org/10.1016/S0065-2407(06)80005-4

Ardila, A., \& Ostrosky, F. (2012). Guía para el diagnóstico neuropsicológico. Florida: American Board of Professional Neuropsychology. Benítez, Y. R., Bringas, M. D., Jiménez-Morales, R. M., \& Macías, V. E. F. (2015). Normas cubanas del instrumento neuropsicológico Luria Inicial para niños preescolares 4-6 años . Cuadernos de Neuropsicología Panamerican Journal of Neuropshychology, 9(2), 49-70. Retrieved from http://10.0.30.34/cnps/9.2.203

Bryck, R. L., \& Fisher, P. A. (2012). Training the brain: Practical applications of neural plasticity from the intersection of cognitive neuroscience, developmental psychology, and prevention science. American Psychologist, 67(2), 87-100. https://doi.org/10.1037/a0024657

Casari, L., \& Oros, M. (2013). Una experiencia de evaluación psicológica en una zona rural. Revista Latinoamericana de Ciencia Psicológica, 5(2) 150-158.

Classen, J. (2013). Plasticity. Handbook of Clinical Neurology, 116, 525-534. https://doi.org/10.1016/B978-0-444-53497-2.00041-3

Dundar, H., Béteille, T., Riboud, M., \& Deolalikar, A. (2014). Early childhood development and the role of preschool. World Bank doi:http://dx.doi.org/10.1596/978-1-4648-0160-0_ch4.

Engle, P. L., Black, M. M., Behrman, J. R., Cabral de Mello, M., Gertler, P. J., Kapiriri, L., ... Young, M. E. (2007). Strategies to avoid the loss of developmental potential in more than 200 million children in the developing world. Lancet. https://doi.org/10.1016/S01406736(07)60112-3

Engle, P. L., Fernald, L. C. H., Alderman, H., Behrman, J., O’Gara, C., Yousafzai, A., ... Iltus, S. (2011). Strategies for reducing inequalities and improving developmental outcomes for young children in low-income and middle-income countries. The Lancet, 378(9799), 1339-1353. https://doi.org/10.1016/S0140-6736(11)60889-1

Ferguson, K. T., Cassells, R. C., MacAllister, J. W., \& Evans, G. W. (2013). The physical environment and child development: an international review. International Journal of Psychology, 48(4), 437-468. https://doi.org/10.1080/00207594.2013.804190

Gottlieb, G. (2002). On the epigenetic evolution of species-specific perception : the developmental manifold concept, 17, $1287-1300$.

Gottlieb, G. (2007). Probabilistic epigenesis. Developmental Science, 10(1), 1-11. https://doi.org/DOI: 10.1111/j.1467-7687.2007.00556.x

Heckman, J. J., \& Masterov, D. V. (2007). The productivity argument for investing in young children. In Review of Agricultural Economics (Vol. 29, pp. 446-493). https://doi.org/10.1111/j.1467-9353.2007.00359.x

Hernandez Sampieri, R., Fernandez Collado, C., \& Baptista Lucio, M. del P. (2010). Metodología de la investigación. Metodología de la investigación (10th ed.). México D.F.: McGrawHill. https://doi.org/- ISBN 978-92-75-32913-9

Knickmeyer, R. C., Gouttard, S., Kang, C., Evans, D., Wilber, K., Smith, J. K., ... Gilmore, J. H. (2008). A structural MRI study of human brain development from birth to 2 years. J Neurosci, 28(47), 12176-12182. https://doi.org/10.1523/JNEUROSCI.3479-08.2008। r28/47/12176 [pii]

Lavrik, SIu., Domitrak, S. V., Shprakh V.V. (2011) Correction of central nervous system energy processes in children with the minimal cerebral dysfunction. Zhurnal Nevrologii I Psikhiatrii Imeni S.S. Korsakova 111 (4), 37-41.

Lenroot, R. K., \& Giedd, J. N. (2006). Brain development in children and adolescents: Insights from anatomical magnetic resonance imaging. Neuroscience and Biobehavioral Reviews. https://doi.org/10.1016/j.neubiorev.2006.06.001

Lowe, J., Erickson, S., MacLean, P., Duvall, S., Ohls, R. y Duncan, A. (2014). Associations between maternal scaffolding and executive functioning in 3 and 4 year olds born very low birth weight and normal birth weight. Early Human Development, 90(10), $587-593$. 
doi: 10.1016/j.earlhumdev.2014.07.009.

Lu, C., Black, M. M., \& Richter, L. M. (2016). Articles Risk of poor development in young children in low-income and middle-income countries: an estimation and analysis at the global, regional, and country level. The Lancet Global Health. https:/doi.org/10.1016/ S2214-109X(16)30266-2

Matute, E., Roselli M. y Ardila, A. (2010). Evaluación Neuropsicológica Infantil. En M., Rosselli, E., Matute, A., Ardila. (Ed) Neuropsicología del desarrollo infantil. (pp. 71-118). México: El Manual Moderno.

Ministerio de Educación Nacional, Departamento Administrativo Nacional de Estadística y Universidad Nacional de Colombia. (2011) Convenio Interadministrativo No. 1551 de 2009 suscrito entre el Ministerio de Educación Nacional, Departamento Administrativo Nacional de Estadistica - DANE y la Universidad Nacional de Colombia. Recuperado de http://www.mineducacion.gov.co/1621/ articles-293674_archivo_pdf_hogares.pdf

Nelson, C. A., Furtado, E. A., Fox, N. A., \& Zeanah, C. H. (2009). The deprived human brain. American Scientist, 97(3), 222-229. https://doi.org/10.1511/2009.78.222

Parra-Pulido, J. H., Rodríguez-Barreto, L. C., \& Chinome-Torres, J. D. (2016). Evaluación de la madurez neuropsicológica infantil en preescolares. Revista Universidad y Salud, 18(1), 126-137. Retrieved from http://www.scielo.org.co/pdf/reus/v18n1/v18n1a13.pdf

Parra Pulido, J. H., Rodríguez Barreto, L. C., \& Chinome Torres, J. D. (2015). Relación entre peso al nacer y madurez neuropsicológica en preescolares de Tunja (Colombia). Pensamiento Psicológico, 13(2), 65-77. https://doi.org/10.11144/Javerianacali.PPSI13-2.rpnm

Pearce, M., Mann, K., Singh, G., \& Sayers, S. (2014). Birth weight and cognitive function in early adulthood: the Australian aboriginal birth cohort study. J Dev Orig Health Dis, 5(03), 240-247. http://dx.doi.org/10.1017/s2040174414000063

Pollak, S. D., Nelson, C. A., Schlaak, M. F., Roeber, B. J., Wewerka, S. S., Wiik, K. L., ... Gunnar, M. R. (2010). Neurodevelopmental effects of early deprivation in postinstitutionalized children. Child Development, 81(1), 224-236. https://doi.org/10.1111/j.14678624.2009.01391.x

Portellano J, García J (2014). Neuropsicología de la atención, las funciones ejecutivas y la memoria. 1 ed. Madrid, España: Editorial síntesis

Portellano, J. A., Mateos, R., \& Martínez-Arias, R. (2012). Cuestionario de Madurez Neuropsicológica Escolar (CUMANES). Madrid: TEA Ediciones.

Portellano Pérez, J. A., Mateos Mateos, R., Martínez Arias, R., Tapia Pavón, A., \& Granados García-Tenorio, M. J. (2000). Cuestionario de madurez neuropsicológica infantil : CUMANIN. TEA. Retrieved from https://dialnet.unirioja.es/servlet/libro?codigo=219925

Ramírez, V., Llamas-Salguero, F., \& López-Fernández, V. (2017). Relación Entre El Desarrollo Neuropsicológico Y La Creatividad En Edades Tempranas . International Journal of Humanities and Social Science Invention, 6(1), 34-40. Retrieved from http://www. ijhssi.org/papers/v6(1)/Version-3/F0601033440.pdf

Rees, P., Booth, R., \& Jones, A. (2016). The emergence of neuroscientific evidence on brain plasticity: Implications for educational practice. Educational \& Child Psychology, 33(1), 8-19.

Schwenck, C., Mergenthaler, J., Keller, K., Zech, J., Salehi, S., Taurines, R., ... Freitag, C. M. (2012). Empathy in children with autism and conduct disorder: Group-specific profiles and developmental aspects. Journal of Child Psychology and Psychiatry and Allied Disciplines. https://doi.org/10.1111/j.1469-7610.2011.02499.x

Sharma, N., Classen, J., \& Cohen, L. G. (2013). Neural plasticity and its contribution to functional recovery. Handbook of Clinical Neurology, 110, 3-12. https://doi.org/10.1016/B978-0-444-52901-5.00001-0

Shonkoff, J. P., Richter, L., van der Gaag, J., \& Bhutta, Z. A. (2012). An Integrated Scientific Framework for Child Survival and Early Childhood Development. PEDIATRICS, 129(2), e460-e472. https://doi.org/10.1542/peds.2011-0366

Shonkoff, Jack P., Boyce, W. T., \& McEwen, B. S. (2009). Neuroscience, Molecular Biology, and the Childhood Roots of Health Disparities. JAMA, 301(21), 2252. https://doi.org/10.1001/jama.2009.754

Shonkoff, Jack P. (2011). Protecting Brains, Not Simply Stimulating Minds. Science, 333(6045), 982-983. https://doi.org/10.1126/science.1206014

Schwenck, C., Mergenthaler, J., Keller, K., Zech, J., Salehi, S., Taurines, R., Romanos, M., Schecklmann, M., Schneider, W., Warnke, A. and Freitag, C.M. (2012), Empathy in children with autism and conduct disorder: group-specific profiles and developmental aspects. Journal of Child Psychology and Psychiatry, 53: 651-659. doi:10.1111/j.1469-7610.2011.02499.x

Tornimbeni, S., Pérez, E., Olaz, F., de Kohan, N. C., Fernández, A., \& Cupani, M. (2008). Introducción a la psicometría. Paidós Buenos Aires.

Zamora Crespo, B., \& García-Navarro, C. (2014). Pruebas neuropsicológicas en pediatría. Anales de Pediatría Continuada, 12(04), 191-197. Retrieved from http://www.apcontinuada.com/es/pruebas-neuropsicologicas-pediatria/articulo/90339622/

Zeanah, C. H., Egger, H. L., Smyke, A. T., Nelson, C. A., Fox, N. A., Marshall, P. J., \& Guthrie, D. (2009). Institutional rearing and psychiatric disorders in Romanian preschool children. American Journal of Psychiatry, 166(7), 777-785. https://oi.org/10.1176/ appi.ajp.2009.08091438 


\section{ANEXOS}

Baremos para niños de 7 años en el índice de desarrollo neuropsicológico

\begin{tabular}{|c|c|c|c|c|c|}
\hline $\begin{array}{l}\text { Suma de puntuaciones } \\
\text { de transformación }\end{array}$ & Puntuación típica & Percentil & $\begin{array}{l}\text { Suma de puntuaciones } \\
\text { de transformación }\end{array}$ & $\begin{array}{l}\text { Puntuación } \\
\text { típica }\end{array}$ & Percentil \\
\hline 28 & 74,00 & 4,00 & 45,00 & 103,00 & 58,00 \\
\hline 31,18 & 78,00 & 7,00 & 45,39 & 104,00 & 61,00 \\
\hline 33,00 & 80,00 & 9,00 & 45,64 & 105,00 & 63,00 \\
\hline 34,13 & 82,00 & 12,00 & 46,00 & 106,00 & 66,00 \\
\hline 36,00 & 85,00 & 16,00 & 47,00 & 107,00 & 68,00 \\
\hline 37,00 & 86,00 & 18,00 & 47,73 & 108,00 & 70,00 \\
\hline 38,30 & 87,00 & 19,00 & 48,00 & 109,00 & 73,00 \\
\hline 38,56 & 88,00 & 21,00 & 48,62 & 110,00 & 75,00 \\
\hline 39,00 & 89,00 & 23,00 & 48,76 & 111,00 & 77,00 \\
\hline 39,70 & 90,00 & 25,00 & 49,00 & 113,00 & 81,00 \\
\hline 40,00 & 91,00 & 27,00 & 49,14 & 114,00 & 82,00 \\
\hline 41,00 & 92,00 & 30,00 & 49,64 & 116,00 & 86,00 \\
\hline 42,00 & 93,00 & 32,00 & 50,00 & 117,00 & 87,00 \\
\hline 42,30 & 94,00 & 34,00 & 53,00 & 118,00 & 88,00 \\
\hline 42,53 & 95,00 & 37,00 & 54,29 & 120,00 & 91,00 \\
\hline 43,00 & 96,00 & 39,00 & 55,00 & 121,00 & 92,00 \\
\hline 43,74 & 98,00 & 45,00 & $\geq 56,98$ & $\geq 124,00$ & 99,00 \\
\hline 44,00 & 99,00 & 47,00 & & & \\
\hline 44,61 & 100,00 & 50,00 & & & \\
\hline 44,80 & 101,00 & 53,00 & & & \\
\hline
\end{tabular}

Baremos para niños de 8 años en el índice de desarrollo neuropsicológico

\begin{tabular}{llllll}
\hline $\begin{array}{l}\text { Suma de puntuaciones de } \\
\text { transformación }\end{array}$ & Puntuación típica & Percentil & $\begin{array}{l}\text { Suma de puntuaciones } \\
\text { de transformación }\end{array}$ & $\begin{array}{l}\text { Puntuación } \\
\text { típica }\end{array}$ & Percentil \\
\hline 35,86 & & & & \\
36,00 & 74,00 & 1,1 & 53,00 & 104,00 & 58,7 \\
37,00 & 76,00 & 3,3 & 53,71 & 105,00 & 63,0 \\
38,18 & 77,00 & 5,4 & 54,32 & 106,00 & 65,2 \\
39,51 & 80,00 & 6,5 & 55,27 & 108,00 & 67,4 \\
40,00 & 83,00 & 9,8 & 55,53 & 109,00 & 69,6 \\
40,32 & 84,00 & 12,0 & 56,00 & 110,00 & 71,7 \\
42,83 & 85,00 & 14,1 & 56,23 & 111,00 & 72,8 \\
43,00 & 87,00 & 15,2 & 56,80 & 113,00 & 79,3 \\
43,29 & 88,00 & 20,7 & 57,00 & 116,00 & 80,4 \\
44,00 & 90,00 & 23,9 & 57,16 & 117,00 & 84,8 \\
44,48 & 91,00 & 28,3 & 58,00 & 118,00 & 88,0
\end{tabular}


Alejandro Pineda-Roa

\begin{tabular}{llllll}
45,45 & 93,00 & 33,7 & 58,83 & 121,00 & 90,2 \\
47,00 & 94,00 & 35,9 & 60 & 124,00 & 92,4 \\
47,51 & 95,00 & 37,0 & 60,22 & 132,00 & 93,5 \\
48,00 & 96,00 & 42,4 & 62,00 & 134,00 & 94,6 \\
50,00 & 97,00 & 46,7 & $\geq 62,54$ & 139,00 & 99,0 \\
50,83 & 98,00 & 48,9 & & \\
51,00 & 99,00 & 50,0 & & \\
52,02 & 101,00 & 54,3 & & \\
52,91 & 103,00 & 57,6 & & \\
\hline
\end{tabular}

Baremos para niños de 9 años en el índice de desarrollo neuropsicológico

\begin{tabular}{|c|c|c|}
\hline Suma de puntuaciones de transformación & Puntuación típica & Percentil \\
\hline 40,00 & 76,00 & 1,4 \\
\hline 48,00 & 83,00 & 4,1 \\
\hline 49,00 & 84,00 & 6,8 \\
\hline 50,00 & 85,00 & 14,9 \\
\hline 52,00 & 86,00 & 20,3 \\
\hline 52,41 & 87,00 & 23,0 \\
\hline 52,86 & 88,00 & 25,7 \\
\hline 52,90 & 90,00 & 27,0 \\
\hline 53,00 & 91,00 & 31,1 \\
\hline 54,00 & 92,00 & 35,1 \\
\hline 54,26 & 93,00 & 37,8 \\
\hline 54,86 & 94,00 & 41,9 \\
\hline 55,00 & 95,00 & 44,6 \\
\hline 56,00 & 96,00 & 50,0 \\
\hline 58,00 & 97,00 & 55,4 \\
\hline 59,00 & 98,00 & 59,5 \\
\hline 59,06 & 99,00 & 63,5 \\
\hline 60,00 & 100,00 & 68,9 \\
\hline 60,02 & 102,00 & 71,6 \\
\hline 60,96 & 104,00 & 77,0 \\
\hline 61,00 & 105,00 & 78,4 \\
\hline 62,56 & 106,00 & 82,4 \\
\hline 63,00 & 107,00 & 85,1 \\
\hline 63,40 & 108,00 & 86,5 \\
\hline 63,53 & 110,00 & 87,8 \\
\hline 64,00 & 115,00 & 90,5 \\
\hline 64,12 & 117,00 & 93,2 \\
\hline 65,66 & 118,00 & 97,3 \\
\hline$\geq 66,00$ & 124,00 & $>99$ \\
\hline
\end{tabular}


Baremos para niños de 10 años en el índice de desarrollo neuropsicológico

\begin{tabular}{|c|c|c|}
\hline Suma de puntuaciones de transformación & Puntuación típica & Percentil \\
\hline 38,60 & 64,00 & 2,6 \\
\hline 40,20 & 68,00 & 14,5 \\
\hline 44,00 & 69,00 & 17,1 \\
\hline 45,75 & 71,00 & 18,4 \\
\hline 46,00 & 72,00 & 39,5 \\
\hline 47,00 & 73,00 & 40,8 \\
\hline 48,00 & 75,00 & 46,1 \\
\hline 50,00 & 76,00 & 47,4 \\
\hline 51,00 & 77,00 & 50,0 \\
\hline 52,00 & 78,00 & 52,6 \\
\hline 53,00 & 79,00 & 53,9 \\
\hline 55,00 & 80,00 & 57,9 \\
\hline 56,00 & 82,00 & 63,2 \\
\hline 58,00 & 84,00 & 65,8 \\
\hline 58,61 & 85,00 & 67,1 \\
\hline 59,00 & 87,00 & 69,7 \\
\hline 59,07 & 88,00 & 73,7 \\
\hline 60,00 & 89,00 & 76,3 \\
\hline 60,08 & 91,00 & 78,9 \\
\hline 60,61 & 93,00 & 82,9 \\
\hline 61,00 & 95,00 & 85,5 \\
\hline 61,21 & 96,00 & 89,5 \\
\hline 62,08 & 97,00 & 90,8 \\
\hline 62,18 & 98,00 & 92,1 \\
\hline 63,56 & 99,00 & 94,7 \\
\hline 65,00 & 100,00 & 96,1 \\
\hline 65,52 & 102,00 & 97,4 \\
\hline 66,00 & 103,00 & 98,7 \\
\hline$\geq 69,00$ & $\geq 106,00$ & $\geq 99$ \\
\hline
\end{tabular}


Julian David Chinome Torres, Lucía Carlota Rodríguez Barreto \& Carlos

Alejandro Pineda-Roa

Baremos para niños de 11 años en el índice de desarrollo neuropsicológico

\begin{tabular}{|c|c|c|}
\hline Suma de puntuaciones de transformación & Puntuación típica & Percentil \\
\hline 47,00 & 61,00 & 1,3 \\
\hline 53,56 & 63,00 & 3,9 \\
\hline 55,67 & 65,00 & 7,9 \\
\hline 58,00 & 69,00 & 11,8 \\
\hline 58,27 & 70,00 & 14,5 \\
\hline 58,71 & 74,00 & 18,4 \\
\hline 60,00 & 76,00 & 19,7 \\
\hline 61,39 & 77,00 & 21,1 \\
\hline 63,00 & 78,00 & 25,0 \\
\hline 64,00 & 80,00 & 27,6 \\
\hline 64,07 & 83,00 & 36,8 \\
\hline 64,24 & 83,13 & 40,8 \\
\hline 64,55 & 84,00 & 43,4 \\
\hline 69,00 & 86,00 & 47,4 \\
\hline 70,93 & 88,00 & 48,7 \\
\hline 71,00 & 89,00 & 51,3 \\
\hline 72,00 & 90,00 & 53,9 \\
\hline 72,57 & 91,00 & 61,8 \\
\hline 72,64 & 93,00 & 64,5 \\
\hline 73,00 & 95,00 & 68,4 \\
\hline 73,24 & 96,00 & 71,1 \\
\hline 74,00 & 97,00 & 72,4 \\
\hline 74,34 & 98,00 & 76,3 \\
\hline 74,67 & 99,00 & 77,6 \\
\hline 75,00 & 100,00 & 82,9 \\
\hline 76,00 & 102,00 & 88,2 \\
\hline 76,26 & 103,00 & 89,5 \\
\hline 76,29 & 104,00 & 90,8 \\
\hline 77,00 & 105,00 & 92,1 \\
\hline 77,60 & 107,00 & 96,1 \\
\hline 78,35 & 108,00 & 97,4 \\
\hline$\geq 78,39$ & $\geq 111,00$ & $\geq 99$ \\
\hline
\end{tabular}

